\title{
Local-overall flexural interaction of pinned-ended thin-walled I-section columns
}

\begin{abstract}
The structural performance of thin-walled compression members are subject to the effects of local buckling, interaction between buckling modes, loading end conditions and material yielding and that due to these effects the compressive carrying capability of thin-walled members can be significantly diminished. This paper employs the finite element simulation to examine the local-overall flexural interaction response of pinned-ended thin-walled I-section columns that covers the complete compressed loading history from the onset of elastic local buckling through the nonlinear elastic and elasto-plastic post-buckling interactive phases of behaviour to final collapse and unloading. A detailed account of the growth and redistribution of stresses on the surfaces is given in the paper. Pinned-ended conditions means, of course, simply supported conditions at the column ends with respect to global rotations and the ends of the constituent plates of the cross-section can be treated as either locally rotationally constrained or locally rotationally free. The numerical simulations take into account the influence of material nonlinearity and geometrical imperfections on the compressive ultimate failures of the sections, however, the study is limited to the interaction of local buckling with overall flexural bending as well as locally rotationally constrained condition. This paper shows that the ultimate failure of the columns is related with yielding on the compression sides of the outer surfaces of the section walls at the web, flanges and section junctions mostly located along the length of the columns.
\end{abstract}

Keyword: Finite element analysis; Local-overall flexural interaction; Pinned-ends; Postbuckling; Thin-walled structures 